\title{
Ruggedizing printed circuit boards using a wideband dynamic absorber
}

\author{
V.C. Ho, A.M. Veprik and V.I. Babitsky* \\ Wolfson School of Mechanical and Manufacturing Engineering, Loughborough University, Leicestershire, LE11 \\ $3 T U, U K$
}

\begin{abstract}
The existing approaches to ruggedizing inherently fragile and sensitive critical components of electronic equipment such as printed circuit boards (PCB) for use in hostile industrial and military environment are either insufficient or expensive. This paper addresses a novel approach towards ruggedizing commercial-off-the-shelf PCBs using a miniature wideband dynamic absorber. The optimisation technique used relies on the experimentally measured vibration spectra and complex receptance of the original PCB.

The analytical study and full-scale experiment show that an optimised wideband dynamic absorber with the mass of only $15 \%$ relative to the total mass of the PCB is quite capable of the essential suppression of all relevant resonant responses of the PCB under shock, wideband random and sinusoidal vibration with variable frequency.
\end{abstract}

\section{Introduction}

Modern industrial and military systems rely widely on electronic equipment containing delicate internal components such as printed circuit boards (PCBs). Plug-in PCBs are typically plate-wise structures, which are manufactured from Epoxy Fibreglass, and carry soldered microchips along with lumped components. The dynamic properties of the commercial-off-theshelf [1] unframed PCBs are such that their stiffness and damping are inherently low [2]. During operation, these delicate electronic components encounter exposure to harsh environmental conditions including shocks, wideband random and swept sine vibration with essential energy content in the wide frequency range from 0 up to $2000 \mathrm{~Hz}[3,4]$. The resulting dynamic responses developed by such PCBs might be exces-

${ }^{*}$ Corresponding author: V.I. Babitsky, Wolfson School of Mechanical and Manufacturing Engineering, Loughborough University, Loughborough, Leicestershire, LE11 3TU, UK. Tel.: +44 01509 227503; Fax: +44 01509 223934; E-mail: V.I.Babitsky@ lboro.ac.uk. sive, thus resulting in failure of the above microchips, lumped components, wiring, soldered joints, etc.

Using dampening sandwich-wise strips allows limited control of the dynamic response of the PCB [2,5], but not to an extent which is required by typical milspec requirements. Since the above strips are adding not only damping but also mass to the original structure, the resulting loss factor cannot be increased significantly. Therefore, since the overall response of dynamic system under random wideband excitation is reciprocal to the above loss factor [6], the attainable performance is not very impressive.

The ruggedizing of the PCB by using stiffening ribs, frames or sandwich-wise structures, as practised in standard military designs $[2,7,8]$, is aimed primarily at increasing the resonant frequencies of the PCB to above the excitation range. The obvious pitfall of this approach is an essential increase in the overall weight, dimension and price, along with a reduction of the useful surface available for the placing of electronic components and wiring.

The authors of [9] considered an application of the tuned dynamic absorber for vibration control of PCB undergoing sinusoidal excitation with constant fre- 
quency. However, in many installations for military and industrial electronic equipment, wideband vibration is of primary concern. Typical military specifications for the testing of this type of equipment $[3,10]$ have placed heaviest emphasis on wideband random vibration, tailored to the actual application. Under shock, wideband random or swept sine excitation the above undamped dynamic absorber can make matters worse, due to excitation of resonant responses, and even cause damage, which would not otherwise happen.

A typical damped dynamic absorber is an auxiliary lightly weighted mass-spring-damper attachment mounted upon a lightly damped primary dynamic structure with the purpose of close control of resonant phenomena under wideband loading. For the best performance, visco-elastic properties of the dynamic absorber should be carefully "tuned" in accordance with the excitation type and dynamical properties of the above primary structure.

In [11], the first attempt to design a dynamic absorber for the optimal suppression of a resonant response of an undamped primary single-degree-freedom (SDOF) structure under forced swept sine excitation was carried out. The authors of [12] presented an expression for optimum absorber parameters for undamped SDOF primary systems, under given harmonic and white noise random force excitations. In [13], the author obtained general formulae for the optimum damper in applications for vibration suppression of an undamped SDOF primary system under given white noise random force excitation. In [14], the influence of damping in the primary system on the optimum absorber parameters was investigated. The authors of [15] addressed the problem of optimal design of a dynamic absorber as applied to a vibration control of an SDOF system under the force and base-induced excitation. In [16], the optimisation of a dynamic absorber for the vibration suppression of the MDOF system subjected to random force input with dominant frequency was addressed. In [17], the authors proposed an efficient optimal design algorithm to minimise the vibratory response of MDOF systems under given sinusoidal excitation over several frequencies. In [18], an effective approach to the optimal design of a dynamic absorber as applied to dynamic structures subjected to the action of a known forced excitation was recently developed. The substructure coupling technique used relies on knowledge of a set of complex frequency response functions for the primary sub-system and for the dynamic absorber.

There are obvious drawbacks to the above known approaches. Evidently, the optimal design of the dy- namic absorber should be based not only on the dynamic model of the primary mechanical structure but also, and primarily, upon the exact knowledge of excitation distribution in spatial and time (frequency) domains. Such information is obtainable only in the simplest cases of academic study; in the majority of realworld applications, this data is simply not available and, hence, rough and often unjustified assumptions are used.

The author of [13] expressed the vibration response of the system equipped with a vibration absorber through the original dynamic response and the local receptance of the unmodified primary mechanical structure and dynamic properties of the dynamic absorber. The obvious advantage of this approach is that detailed information on the excitation distribution in time and spatial domains and corresponding frequency response characteristics of the original mechanical system is not required at all.

In the present paper, the authors are attempting to further accommodate the above idea for optimal design of a wideband dynamic absorber with application to vibration protection of commercial-off-the-shelf PCBs subjected to harsh environmental vibration.

\section{Dynamic model of PCB with dynamic absorber}

We assume that the point of the PCB where the critical failure takes place (further - critical point) is known from previous experience. We also assume at this stage that by the design of the PCB and the entire electronic package it is possible to mount the dynamic absorber adjacent to the above critical point.

Figure 1 shows the model of the PCB with a dynamic absorber attached. As mentioned in the introduction, the exact knowledge of the excitation (base vibration, $z(t)$, in this instance) is not required. In Fig. 1, $u\left(x^{\prime}, y^{\prime}, t\right)$ is the absolute motion of the critical point of the PCB, $\left(x^{\prime}, y^{\prime}\right)$, where the absorber is mounted and $q(t)$ is the motion of the dynamic absorber mass relative to its mounting point.

Further $\mathcal{Z}[*]$ denotes the operator of the Laplace transform. Under the assumption that the dynamic system under consideration is linear, the principle of superposition and the Laplace calculus yield the motion of the critical point of the modified system in the form:

$$
\begin{gathered}
\mathcal{Z}\left[u\left(x^{\prime}, y^{\prime}, t\right)\right]=\mathcal{Z}\left[u_{0}\left(x^{\prime}, y^{\prime}, t\right)\right] \\
-H\left(x^{\prime}, x^{\prime} ; y^{\prime}, y^{\prime} ; s\right) \mathcal{Z}[R(t)],
\end{gathered}
$$




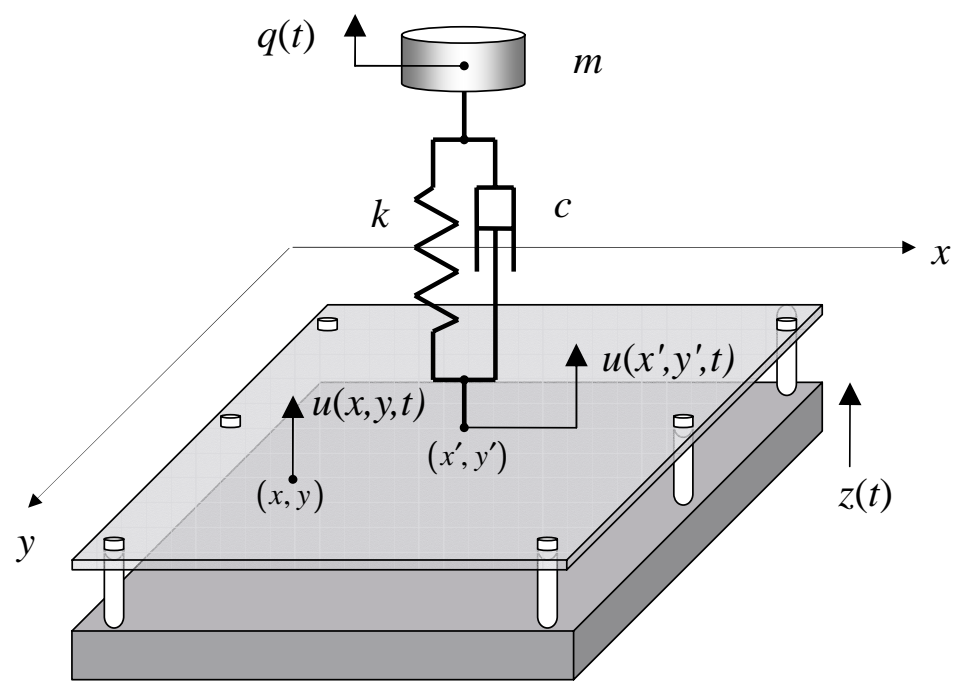

Fig. 1. Dynamic model of the PCB with dynamic absorber.

where $s$ is a complex (Laplace) variable; the transfer function, $H\left(x^{\prime}, x^{\prime} ; y^{\prime}, y^{\prime} ; s\right)$, is the operator of the local receptance of the original system; and $R(t)=$ $c \dot{q}(t)+k q(t)$ is the force of the dynamic reaction, developed due to the relative motion of the absorber and PCB. On the right-hand side of Eq. (1), the first addend represents the "original" motion - the motion of the primary structure under actual excitation with no dynamic absorber attached. The second addend represents the motion of the primary structure, originated by the above force of dynamic reaction, $R(t)$.

The Laplace transform of the equation of motion for the dynamic absorber takes the form:

$$
\begin{gathered}
\mathcal{Z}[m \ddot{q}(t)+c \dot{q}(t)+k q(t)]= \\
\mathcal{Z}\left[-m \frac{\partial^{2} u\left(x^{\prime}, y^{\prime}, t\right)}{\partial t^{2}}\right] .
\end{gathered}
$$

From Eqs (1) and (2), at zero initial conditions we obtain the set of equations:

$$
\begin{aligned}
& U\left(x^{\prime}, y^{\prime}, s\right)=U_{0}\left(x^{\prime}, y^{\prime}, s\right) \\
& -H\left(x^{\prime}, x^{\prime} ; y^{\prime}, y^{\prime} ; s\right)(c s+k) Q(s), \\
& Q(s)=\frac{-m s^{2}}{m s^{2}+c s+k} U\left(x^{\prime}, y^{\prime}, s\right),
\end{aligned}
$$

where the following Laplace transforms are used: $Q(s) \Leftrightarrow q(t), \quad U\left(x^{\prime}, y^{\prime}, s\right) \Leftrightarrow u\left(x^{\prime}, y^{\prime}, t\right)$ and $U_{0}\left(x^{\prime}, y^{\prime}, s\right) \Leftrightarrow u_{0}\left(x^{\prime}, y^{\prime}, t\right)$.

From Eq. (3), the vibration of the critical point of the modified system is found by excluding $Q(s)$, as follows:

$$
\begin{aligned}
& U\left(x^{\prime}, y^{\prime}, s\right)=U_{0}\left(x^{\prime}, y^{\prime}, s\right) \\
& \times \frac{1}{1-H\left(x^{\prime}, x^{\prime} ; y^{\prime}, y^{\prime} ; s\right) \frac{m s^{2}(c s+k)}{m s^{2}+c s+k}} .
\end{aligned}
$$

The formal substitution $s=j \omega$, where $j=\sqrt{-1}$ and $\omega$ is the angular frequency, yields a transition into the frequency domain. By so doing we represent the motion of the critical point of the PCB in the form of the complex spectrum:

$$
\begin{aligned}
& U\left(x^{\prime}, y^{\prime}, j \omega\right)= \\
& \frac{U_{0}\left(x^{\prime}, y^{\prime}, j \omega\right)}{1+H\left(x^{\prime}, x^{\prime} ; y^{\prime}, y^{\prime} ; j \omega\right) \frac{m \omega^{2}(j \omega c+k)}{-m \omega^{2}+j \omega c+k}} .
\end{aligned}
$$

expressed through the absorber parameters, the complex spectrum of the original motion of the critical point and local complex receptance of the primary structure with no absorber attached.

Further, we consider the case of stationary random excitation (see [19] for the reference). From Eq. (5), the response of the composite system, $U\left(x^{\prime}, y^{\prime}, j \omega\right)$, may be formally thought of as a result of the input $U_{0}\left(x^{\prime}, y^{\prime}, j \omega\right)$ Following [19], the appropriate power spectral density (PSD) takes the form

$$
\begin{aligned}
& S\left(x^{\prime}, y^{\prime}, \omega\right)= \\
& \frac{S_{0}\left(x^{\prime}, y^{\prime}, \omega\right)}{\left|1+H\left(x^{\prime}, x^{\prime} ; y^{\prime}, y^{\prime} ; j \omega\right) \frac{m \omega^{2}(c j \omega+k)}{-m \omega^{2}+c j \omega+k}\right|^{2}} .
\end{aligned}
$$

Equation (6) predicts the PSD of absolute vibration of the critical point of the modified PCB based on: the values of the dynamic absorber parameters $m, k$ and 
$c$; the measured PSD of original motion, $S_{0}\left(x^{\prime}, y^{\prime}, \omega\right)$; and complex local receptance, $H\left(x^{\prime}, x^{\prime} ; y^{\prime}, y^{\prime} ; j \omega\right)$ of the original PCB with no absorber attached.

The above technique may also be adapted for prediction of the dynamic response of the arbitrary point of the PCB. This is especially important when the design of a particular PCB or the entire electronic package makes it impossible to place the dynamic absorber at the critical point, or it is required to suppress vibration at a variety of critical points of the PCB by using a single dynamic absorber installed at the most convenient location.

Similar to Eq. (3), to define the motion of the arbitrary point $(x, y)$ of the modified system we use the following set of equations:

$$
\begin{aligned}
& U\left(x^{\prime}, y^{\prime}, s\right)=U_{0}\left(x^{\prime}, y^{\prime}, s\right) \\
& -H\left(x, x^{\prime} ; y, y^{\prime} ; s\right)(c s+k) Q(s), \\
& U(x, y, s)=U_{0}(x, y, s) \\
& -H\left(x^{\prime}, x^{\prime} ; y^{\prime}, y^{\prime} ; s\right)(c s+k) Q(s), \\
& Q(s)=\frac{-m s^{2}}{m s^{2}+c s+k} U\left(x^{\prime}, y^{\prime}, s\right),
\end{aligned}
$$

where the transfer function, $H\left(x, x^{\prime} ; y, y^{\prime} ; s\right)$, is the operator of transient receptance and the Laplace transforms $U(x, y, s) \Leftrightarrow u(x, y, t)$ and $U_{0}(x, y, s) \Leftrightarrow$ $u_{0}(x, y, t)$ are used.

Similarly to Eq. (5), making the transition into the frequency domain yields vibration of the arbitrary point of the modified system in the form of the complex spectrum:

$$
\begin{gathered}
U(x, y, j \omega)=U_{0}(x, y, j \omega)-U_{0}\left(x^{\prime}, y^{\prime}, j \omega\right) \\
\times \frac{H\left(x, x^{\prime} ; y, y^{\prime} ; j \omega\right)}{\frac{-m \omega^{2}+j \omega c+k}{m \omega^{2}(j \omega c+k)}+H\left(x^{\prime}, x^{\prime} ; y^{\prime}, y^{\prime} ; j \omega\right)} .
\end{gathered}
$$

In Eq. (8), the two processes, $U_{0}(x, y, j \omega)$ and $U_{0}\left(x^{\prime}, y^{\prime}, j \omega\right)$, are the correlated original responses of the two different points of the PCB exposed to the actual excitation with no absorber attached. From Eq. (9), the total response of the modified system, $U(x, y, j \omega)$, may be formally thought of as a response of a dynamic system to the correlated inputs $U_{0}(x, y, j \omega)$ and $U_{0}\left(x^{\prime}, y^{\prime}, j \omega\right)$.

Following [19], the appropriate PSD takes the form:

$$
\begin{aligned}
S(x, y, \omega)= & S_{0}(x, y, \omega) \\
& +S_{0}\left(x^{\prime}, y^{\prime}, \omega\right) \times|L(j \omega)|^{2} \\
& -S_{0}\left(x, x^{\prime} ; y, y^{\prime} ; j \omega\right) L(j \omega) \\
& -S_{0}^{*}\left(x, x^{\prime} ; y, y^{\prime} ; j \omega\right) L^{*}(j \omega)
\end{aligned}
$$

where $S_{0}\left(x, x^{\prime} ; y, y^{\prime} ; j \omega\right)$ is the cross-spectrum between the two above mentioned correlated processes $U_{0}\left(x^{\prime}, y^{\prime}, j \omega\right)$ and $U_{0}(x, y, j \omega)$ and

$$
L(j \omega)=\frac{H\left(x, x^{\prime} ; y, y^{\prime} ; j \omega\right)}{\frac{-m \omega^{2}+j \omega c+k}{m \omega^{2}(j \omega c+k)}+H\left(x^{\prime}, x^{\prime} ; y^{\prime}, y^{\prime} ; j \omega\right)} .
$$

is a composite frequency response function. In Eq. (9), $S_{0}^{*}\left(x, x^{\prime} ; y, y^{\prime} ; j \omega\right)$ and $L^{*}(j \omega)$ are the complex conjugates of $S_{0}\left(x, x^{\prime} ; y, y^{\prime} ; j \omega\right)$ and $L(j \omega)$, respectively. These functions may be easily measured using modern dual-channel signal analysers.

\section{Dynamic responses and properties of the original PCB}

\subsection{PSD of absolute acceleration of the original PCB under typical base-induced excitation}

The common practice in industrial and military applications is to mount a PCB using pillars and screws allowing for high-pressure interfaces $[2,10]$. Figure 2(a) shows the schematics of the experimental rig. The typical commercial-off-the-shelf Epoxy Fibre Glass PCB (1)is mounted upon fixture (2), which is attached to the vibration test system (3)(V550 Series, Ling Dynamic System). The miniature accelerometer (4) (Bruel and Kjaer, Type 4393) is mounted upon the fixture and provides a signal for the internal feedback loop for the System Controller (6) (DVC-48, Ling Dynamic System Ltd). The controller is interfaced with Terminal 5. A vibration test system is programmed to provide the closed-loop controllable wide-band random vibration. The Polytec OFV 512 Fibre Interferometer (7) and Polytec OFV 3001 Vibrometer Controller (8) are used to measure the absolute velocity of the PCB. The Signal Analyser (9) (Data Physics Corporation, model DP104-100) provides for the appropriate data acquisition and DSP using terminal (5). This experiment involves using non-contact measurement with the advantages of avoiding unnecessary additional mass or interference to the PCB.

To simulate the dynamic response of a PCB to the actual working environment, the vibration test controller was programmed to reproduce a wide-band random vibration with uniform PSD $0.1 \mathrm{~g}^{2} / \mathrm{Hz}$ in the frequency range $20-2000 \mathrm{~Hz}$ (overall level $14 \mathrm{~g}$ RMS, typical military specification in accordance with [3]). Figure 2(b) shows the experimentally obtained typical PSD of absolute acceleration at the critical point of the original $\mathrm{PCB}$, where the overall level is $70.2 \mathrm{~g}$ rms. From 


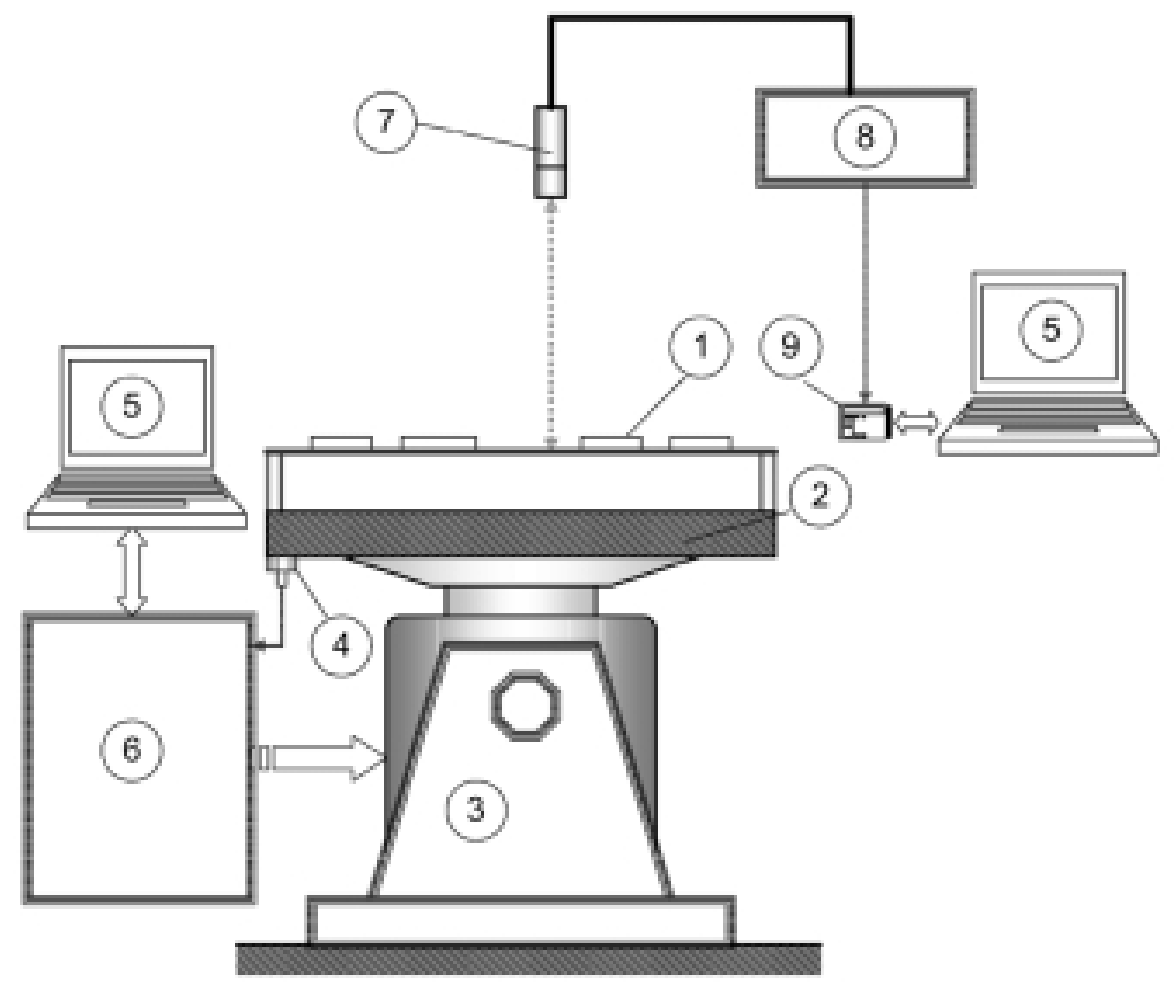

(a)

Experimental rig

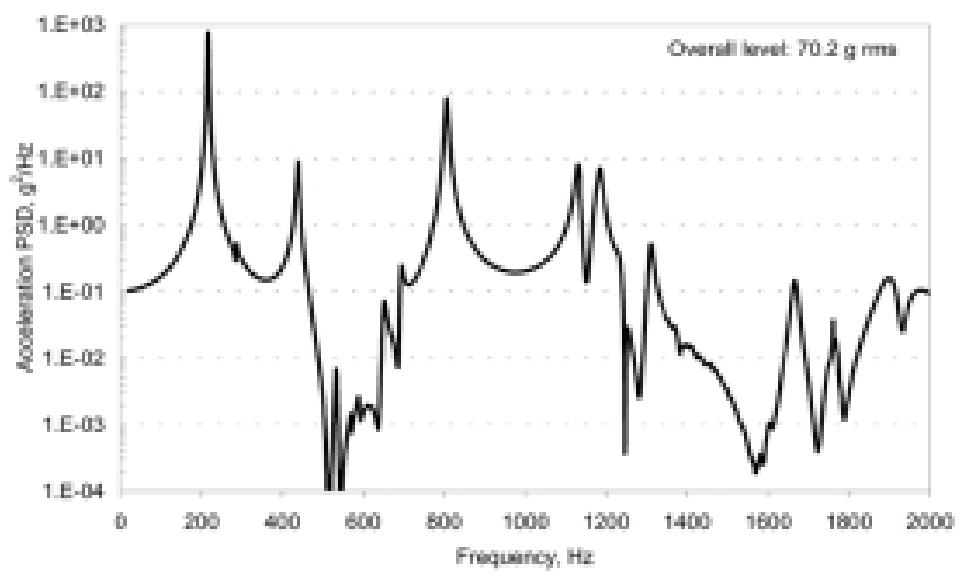

(b)

Experimentally measured PSD of absolute acceleration in the critical point of the original PCB

Fig. 2. Schematics of experimental rig and typical vibration response. a) Experimental rig; b) Experimentally measured PSD of absolute acceleration in the critical point of the original PCB.

Fig. 2(b), the PCB is a multiple-degree-of-freedom system, where the contribution of the higher modes is quite significant. Such a system cannot be analysed as a SDOF system, as proposed, for example, in [2].

\subsection{Complex receptances}

Figure 3(a) shows the experimental rig where the fixture (2) supports the PCB (1) and is rigidly clamped 
to the heavy isolated bench (3). The instrumented impact hammer (4) (Brüel \& Kæer, Type 8203) contains a piezoceramic force transducer (5) providing force excitation to the PCB. Through the charge amplifier (9) (Brüel \& Kjær, Type 2635), the signal of the excitation force is passed to the first channel of Signal Analyser (10.) Simultaneously, the laser vibrometer (7) measures the absolute velocity of PCB. This signal is then passed to the second channel of the Signal Analyser, providing appropriate data acquisition and analysis. Figure 3(b) shows a typical module of the experimentally measured local receptance.

\section{Optimal design of dynamic absorber}

Accumulated fatigue is a common failure mechanism in solder joints and gullwing leads of surface mount assemblies in the electronic packaging industry. Under vibration, the forces of inertia, the magnitudes of which depend upon the local absolute accelerations of the PCB [20], are known to be a major factor contributing to the stresses and strains history, and, therefore, fatigue accumulated at the particular critical points [21].

The failures observed in the electronic components subjected to ergodic random wideband stresses with zero mean value typically follow the conventional failure model of fatigue damage accumulation $[22,23]$ in the form:

$$
\mathbf{D} \sim \sigma_{A}^{b}\left(x^{\prime}, y^{\prime}\right),
$$

where $\mathbf{D}$ is the accumulated fatigue damage, $\sigma_{A}\left(x^{\prime}, y^{\prime}\right)$ is the root mean square (rms) value of the above acceleration

$$
\sigma_{A}\left(x^{\prime}, y^{\prime}\right)=\sqrt{\frac{1}{2 \pi} \int_{0}^{\infty} S\left(x^{\prime}, y^{\prime}, \omega\right) \omega^{4} d \omega}
$$

and $b=9$ is the typical fatigue curve index [24] for the copper or aluminium wiring and soft solders [3,23].

Therefore, following from this, we consider the practical case in which it would be required to minimise the overall rms of the absolute acceleration Eq. (11) at the critical point of the PCB by varying parameters $m, k$ and $c$.

It would be convenient to express the properties of the dynamic absorber in terms of its natural frequency $\frac{1}{2 \pi} \sqrt{\frac{k}{m}}$ and loss factor $\frac{c}{2 \sqrt{m k}}$. To choose the above optimal properties, the numerical procedure has been developed based on Eqs (6) and (7) with experimentally measured data involved (see Figs 3 and 4).
We studied the influence of the mass of the dynamic absorber on its overall performance. For this purpose, we varied the mass of the absorber from $0.01 \mathrm{~kg}$ to $0.1 \mathrm{~kg}$ with increments of $0.01 \mathrm{~kg}$. For each value of mass, we further obtained the natural frequency and loss factor minimising the overall rms response of the above critical point of the PCB. Figure 4(a) shows the dependence of the minimised overall rms level of absolute acceleration on the mass of the dynamic absorber. Figures 4(b) and (c) show the dependencies of the optimised natural frequency and loss factor on the mass of the dynamic absorber, respectively.

From Fig. 4, the higher value of the absorber mass yields the better performance. As far as the weight and space are concerned, we chose the mass of the absorber to be $0.03 \mathrm{~kg}$, thus producing a typical mass ratio of only $15 \%$ relative to the total mass of the PCB.

It is worth noting that choosing the highest mass of the dynamic absorber leads to excessively high values of the required loss factors; these might be problematic to achieve in practice. From the above optimisation procedure, for such a mass chosen, the optimal natural frequency and loss factor of the dynamic absorber should be $184 \mathrm{~Hz}$ and $81 \%$, respectively.

Figure 5 shows a superimposed PSD of absolute acceleration of the original (experiment) and modified (analytical prediction) PCB at the same critical point. From Fig. 5, the influence of the dynamic absorber is that almost all dominating resonances of the original PCB are essentially suppressed; this indicates that the dynamic absorber is mounted at an appropriate position, which does not contain the nodal points of the influential modes. The comparison indicates a 3.2-fold reduction of the overall level from $70.2 \mathrm{~g}$ rms to $22 \mathrm{~g}$ rms.

In practice, however, to design and manufacture the dynamic absorber with such a loss factor within tight mass and space limits is quite a complex engineering task and, thus, the final cost of the entire unit would be unreasonably high. Therefore, the authors further consider a more realistic value of the loss factor, namely, $45 \%$, which might be achieved easily using commercially available vibration mounts. The optimisation procedure was repeated to find a new optimal value of dynamic absorber natural frequency $-209 \mathrm{~Hz}$, corresponding to the above accepted value of the loss factor. Such a dynamic absorber effectively suppresses the overall level of absolute acceleration up to the value of $23.2 \mathrm{~g} \mathrm{rms}$; the degradation in performance is only $4 \%$ as compared with the truly optimal dynamic absorber with loss factor and natural frequency $81 \%$ and $184 \mathrm{~Hz}$. 


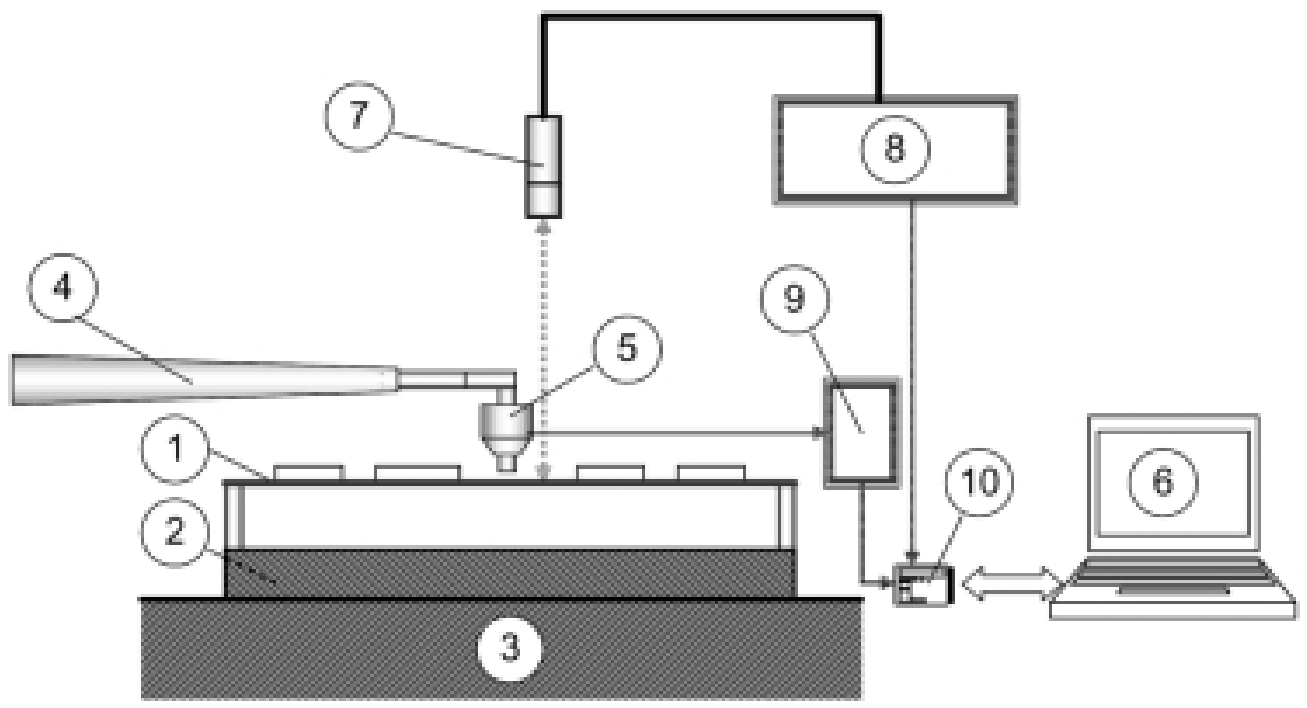

(a)

Experimental rig

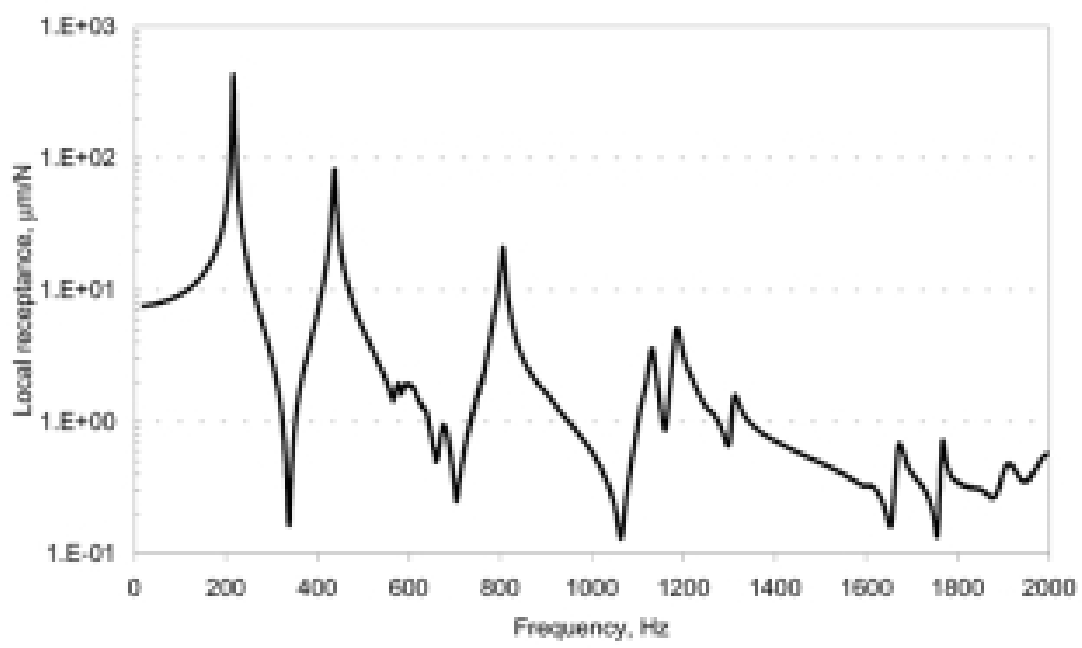

(b)

Module of local reacceptance of PCB

Fig. 3. Schematics of experimental rig and typical module of complex receptance. a) Experimental rig; b) Module of local reacceptance of PCB.

Further, we have considered the optimal design of the vibration absorber mounted at its original location (as above) with properties to suppress vibration at the four additional remote critical points. The calculation, which was based on the appropriate measurements and Eq. (10) with $m=0.03 \mathrm{~kg}$, showed that the originally optimised dynamic absorber yields almost optimal suppression of vibration at the above four critical points. In the experimental section of the article, the original and modified spectra at the above points are shown. The detailed discussion on this result is, however, beyond the scope of the present article.

\section{Sensitivity analysis}

The sensitivity analysis was carried out in the following manner. Firstly, for the chosen mass of the dynamic absorber $(0.03 \mathrm{~kg})$, the loss factor was fixed at $45 \%$, while the natural frequency was varied in the range 50 


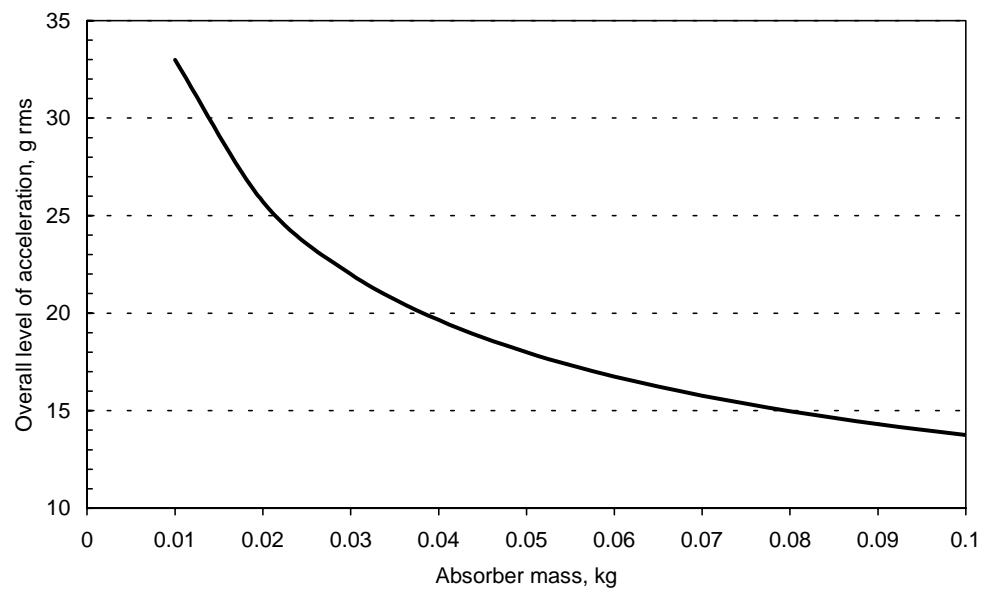

(a) Optimal overall acceleration as a function of dynamic absorber mass

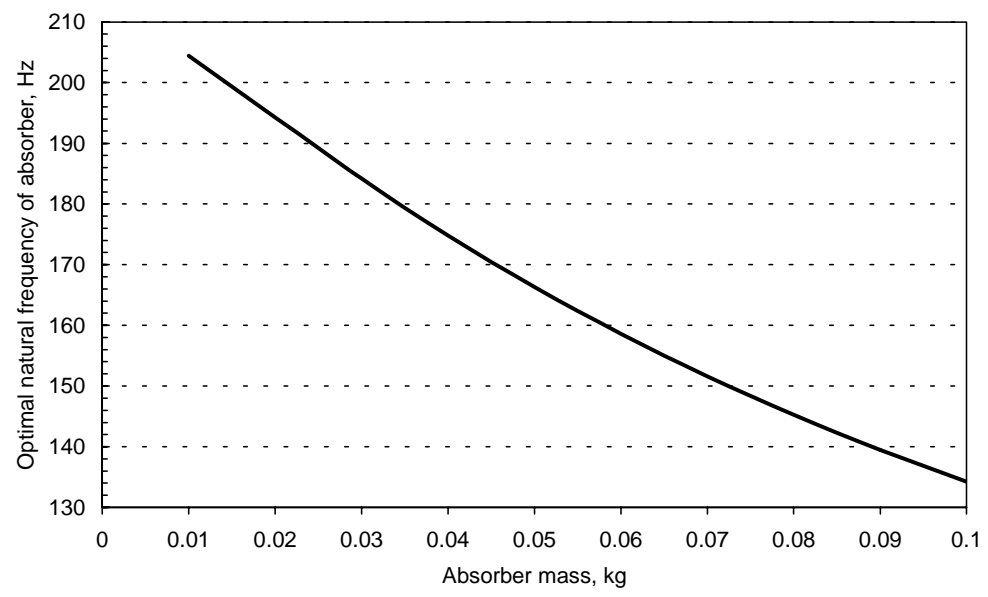

(b) Optimal natural frequency as a function of dynamic absorber mass

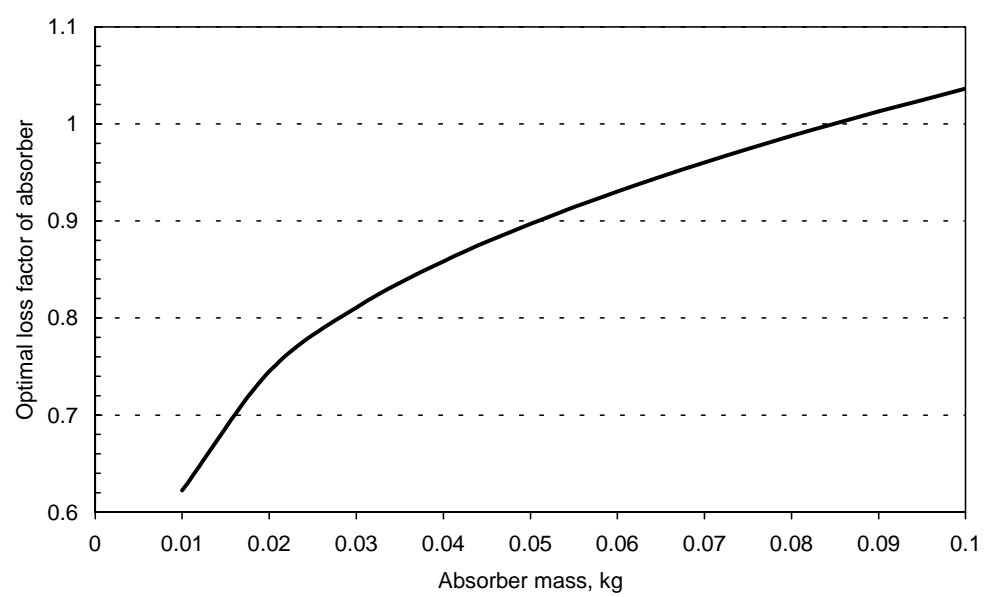

(c) Optimal loss factor as a function of dynamic absorber mass

Fig. 4. Performance and optimal parameters of dynamic absorber at different absorber masses. a) Optimal overall acceleration as a function of dynamic absorber mass; b) Optimal natural frequency as a function of dynamic absorber mass; c) Optimal loss factor as a function of dynamic absorber mass. 


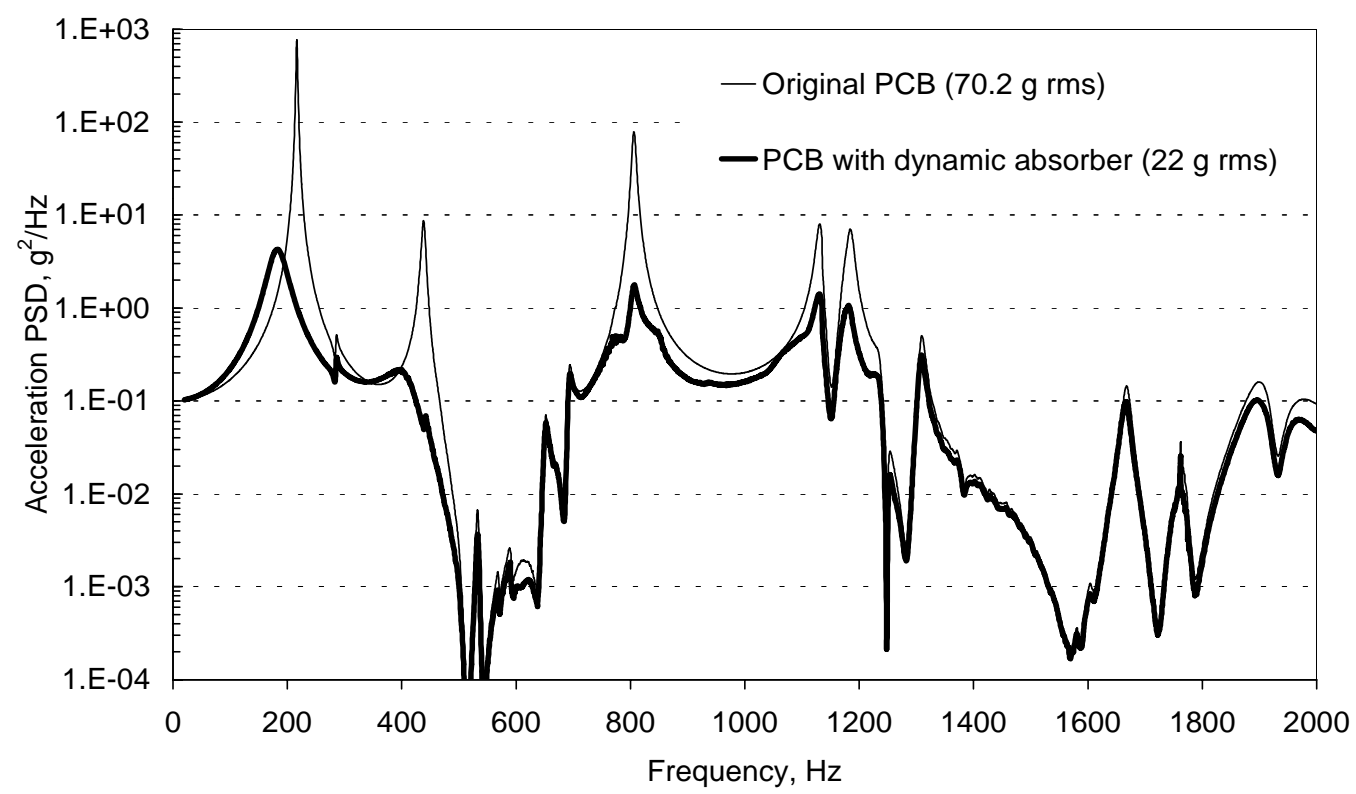

Fig. 5. Performance of the optimal dynamic absorber: PSD of absolute acceleration of original and modified PCB in the control point.

to $500 \mathrm{~Hz}$. The dependence of the obtained overall rms acceleration on the value of the natural frequency is shown in Fig. 6(a). Secondly, for the same mass of the dynamic absorber, the optimal natural frequency, $209 \mathrm{~Hz}$, was fixed while the loss factor was varied in the range from $10 \%$ to $100 \%$. The dependence of the overall rms acceleration on the value of the loss factor is shown in Fig. 6(b).

From Fig. 6, a reasonably small deviation of the values of stiffness and damping of the dynamic absorber from their optimal values due to technological tolerances, temperature variation, ageing, etc. does not cause essential degradation in the overall performance.

\section{Experimentation on attained performance under wideband random vibration}

\subsection{Dynamic properties of dynamic absorber}

In application, the dynamic absorber, as schematically shown in Figs 7(a) and (b), consists of the commercially available Barry Control (see http://www. barrymounts.com) wire mesh bushing (1) carrying the washer (2), which is manufactured from Tungsten for compactness. The mass of the above washer is $0.03 \mathrm{~kg}$; the adjustment of frequency and loss factor of the dynamic absorber relies on squeezing the above wire mesh bushing using screw (3) and nut (4). Such a wire mesh bushing is produced of stainless spring steel wire and is, therefore, capable of maintaining the persistent mechanical properties over a wide temperature range and lifetime.

Figure 7(c) shows the module of absolute transmissibility of the developed dynamic absorber. From the SDOF curve fitting, we identified the loss factor and natural frequency to be 0.4 and $200 \mathrm{~Hz}$, respectively. This is fairly close to the desired optimal values.

\subsection{Composite system}

In the following experiment, we measured the overall rms of absolute acceleration of the critical point of the PCB under the wide-band random vibration with uniform PSD $0.1 \mathrm{~g}^{2} / \mathrm{Hz}$ in the frequency range 20$2000 \mathrm{~Hz}$ (overall level $14 \mathrm{~g} \mathrm{RMS}$ ). The schematics of the experimental rig and apparatus are the same as in Fig. 1. Figure 8(a) shows the PCB with the dynamic absorber attached. Figures 8(b) and (c) compare the dynamic responses of the original and modified PCBs in frequency (a) and in time domains (b). These experimental results are in full agreement with the results of the analytical prediction in Fig. 5. From Fig. 8(b), the influence of the dynamic absorber is that almost all resonances of the original PCB are essentially suppressed, as theoretically predicted above. The comparison indicates a 2.9-fold reduction in the overall level from $70.2 \mathrm{~g}$ rms to $23.9 \mathrm{~g}$ rms. From Fig. (c) the peak value of absolute acceleration is reduced by the same factor of 2.9 from $216 \mathrm{~g}$ to $75 \mathrm{~g}$. Using Eq. (10) with $b=9$, 


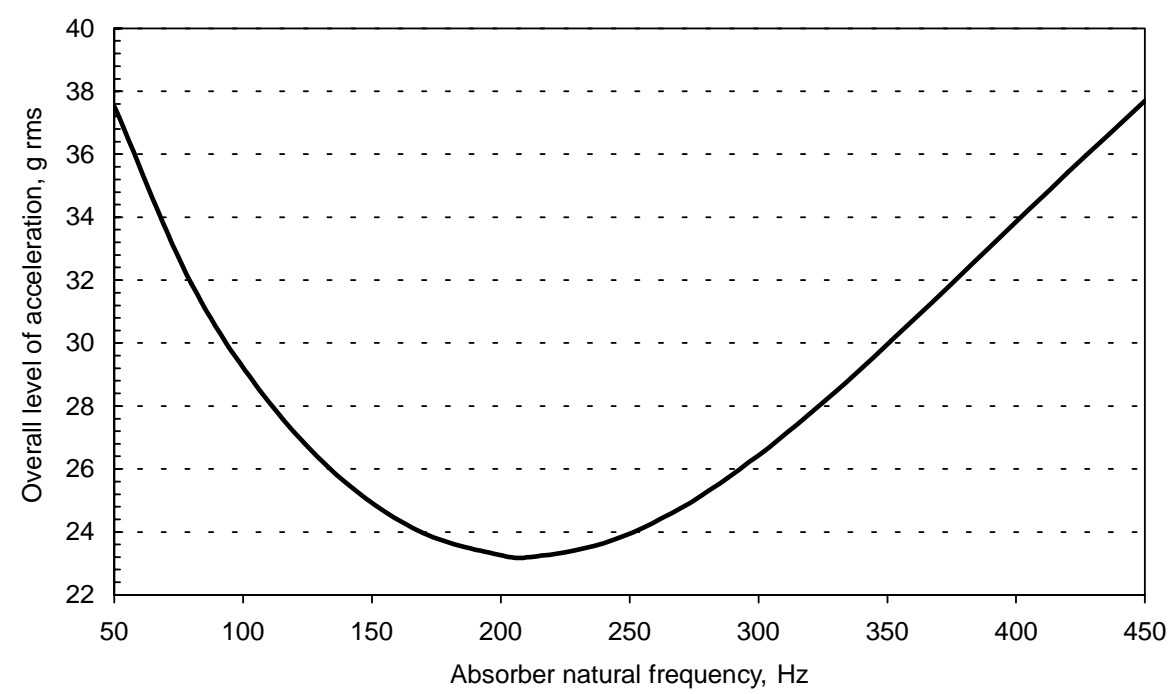

(a) Overall level of acceleration at different natural frequencies

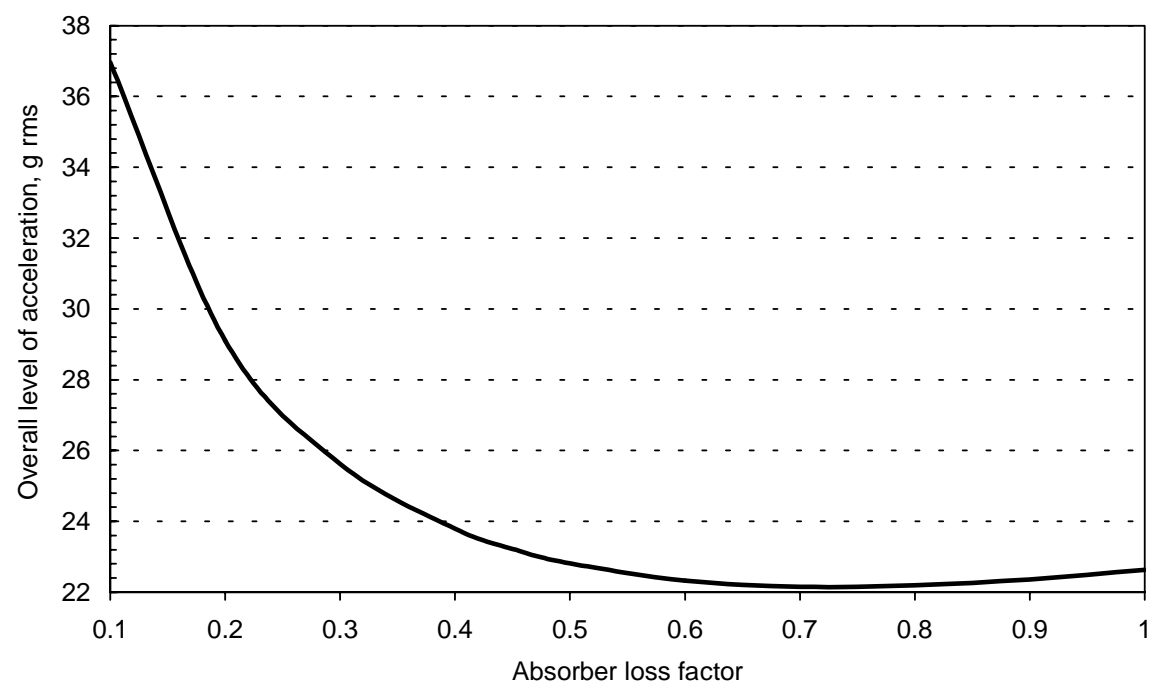

(b) Overall absolute acceleration at different loss factors

Fig. 6. Performance of the dynamic absorber at different natural frequencies and loss factors. a) Overall level of acceleration at different natural frequencies; b) Overall absolute acceleration at different loss factors.

we obtain, for example, that at this particular critical point the accumulated damage undergoes a reduction by a factor of $2.9^{9} \approx 14500$.

Note that the above dynamic absorber essentially suppresses the dynamic response of the entire PCB. Figure 9 shows the superimposed dynamic responses measured at four additional critical points of the PCB before and after the dynamic absorber was mounted. From Fig. 9, the above dynamic absorber provides for almost uniform suppression of PCB vibration. The sup- pression factor, comparing vibration before and after the absorber was installed, varies from 2.9 to 2 .

\section{Experimentation on attained performance under shock and swept sine excitation}

The above dynamic absorber was designed with properties to minimise the overall level of absolute acceleration of the critical point of the PCB under wideband random excitation. However, suppression of other 


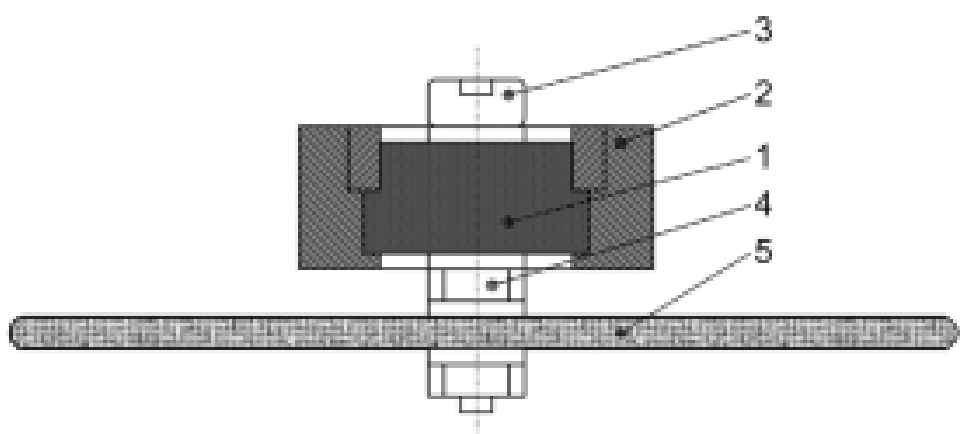

(a) Schematic design of dynamic absorber

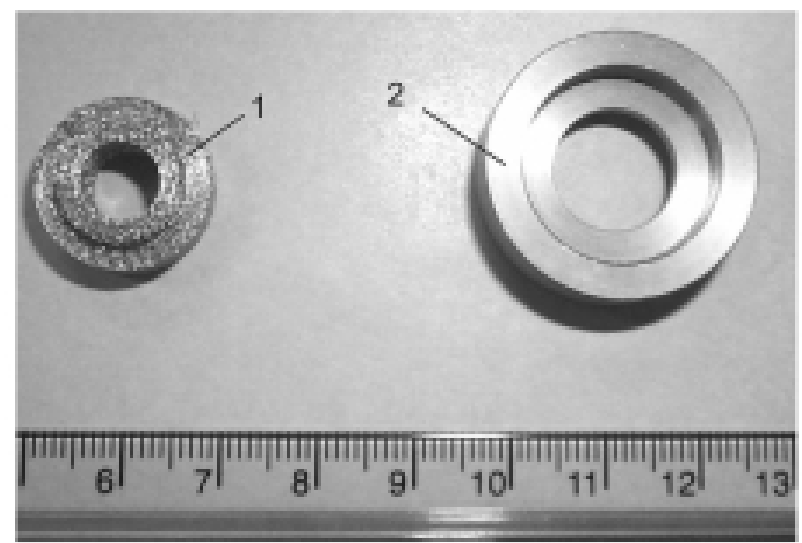

(b) Wire mesh bushing (1) and dynamic absorber washer (2)

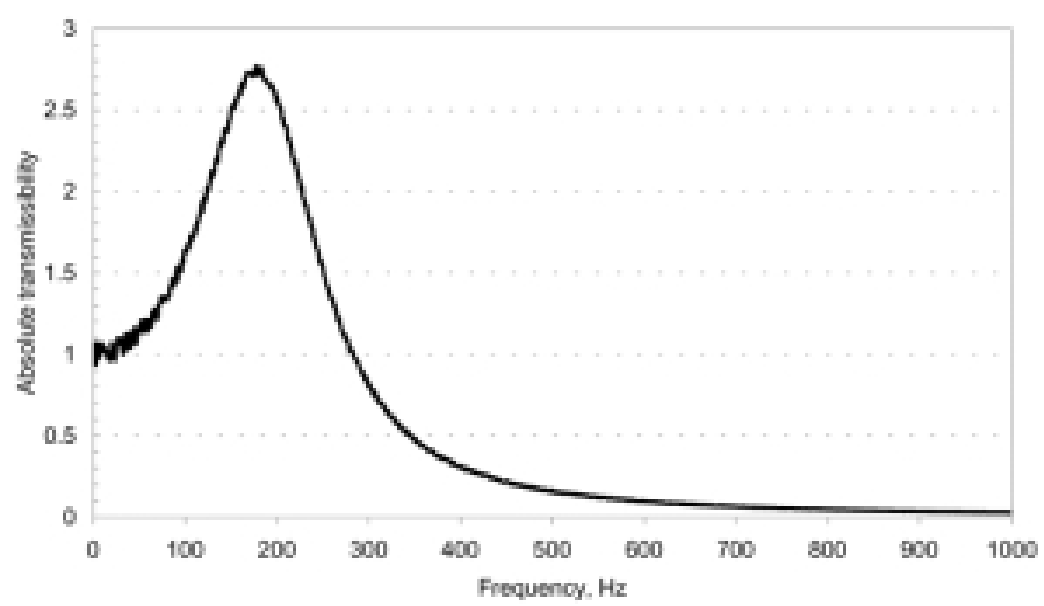

(c) Absolute transmissibility of dynamic absorber

Fig. 7. Design of dynamic absorber. a) Schematic design of dynamic absorber; b) Wire mesh bushing (1) and dynamic absorber washer (2); c) Absolute transmissibility of dynamic absorber.

types of excitation, e.g. shock and swept sine, might also be very important in a variety of applications.

The vibration controller was programmed to reproduce the half-sine shock $200 \mathrm{~g} @ 3$ ms, per [3]. The time history of the shaker acceleration is shown in Fig. 10(a). Figure 10(b) shows superimposed time histories - absolute accelerations of the critical point of the PCB before and after absorber mounting. From 


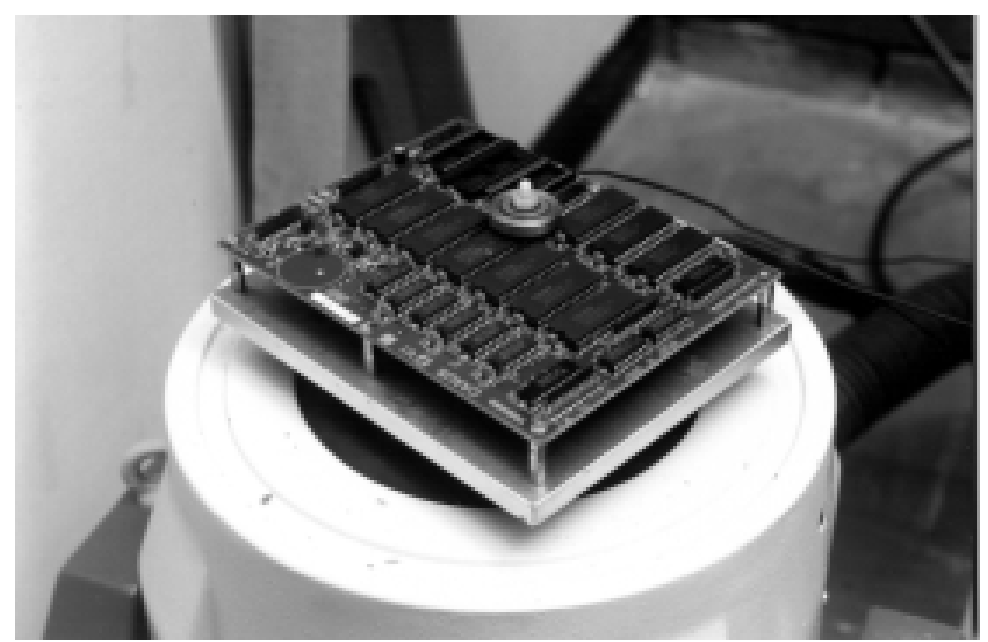

(a) Experimental rig for studying the performance of the dynamic absorber

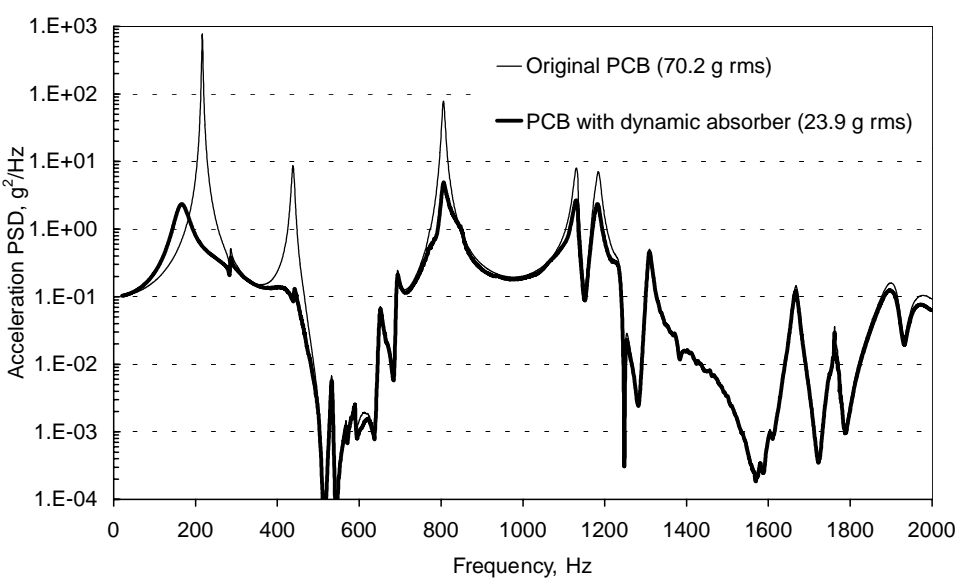

(b) PSD of absolute acceleration in the critical point of original and modified PCB

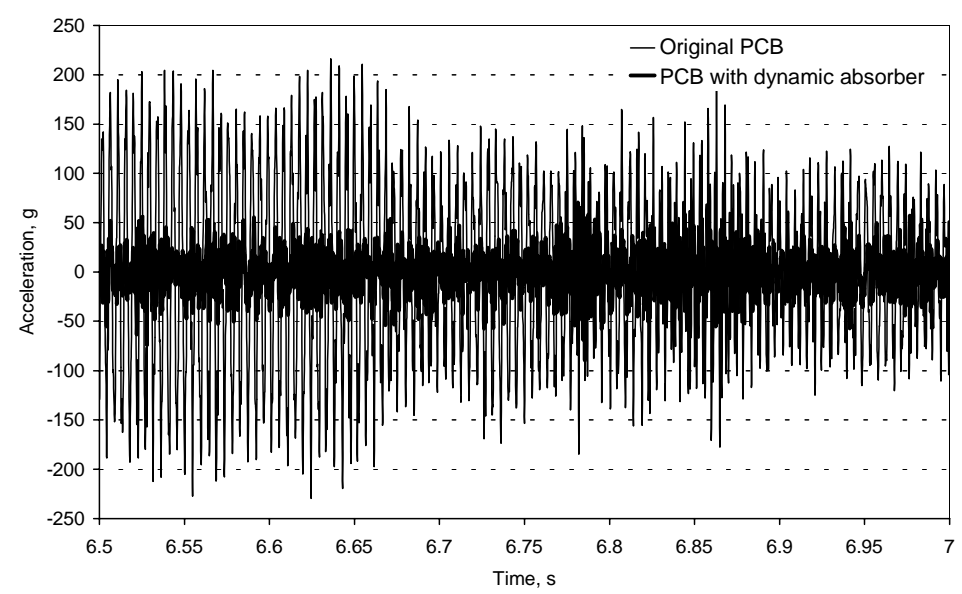

(c) Typical accelerations of the critical point of original and modified PCB

Fig. 8. Performance of dynamic absorber in frequency and time domains. a) Experimental rig for studying the performance of the dynamic absorber; b) PSD of absolute acceleration in the critical point of original and modified PCB; c) Typical accelerations of the critical point of original and modified PCB. 


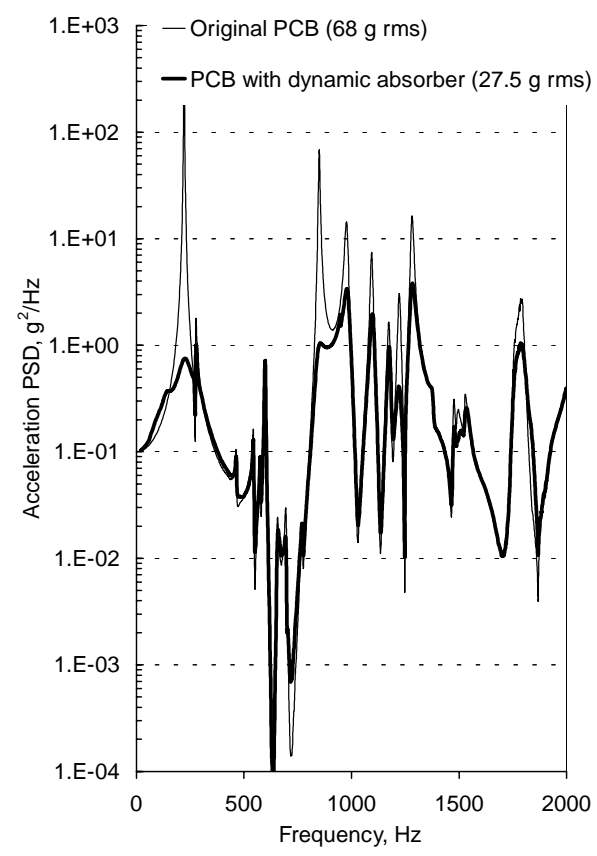

Point (1), suppression factor 2.5.

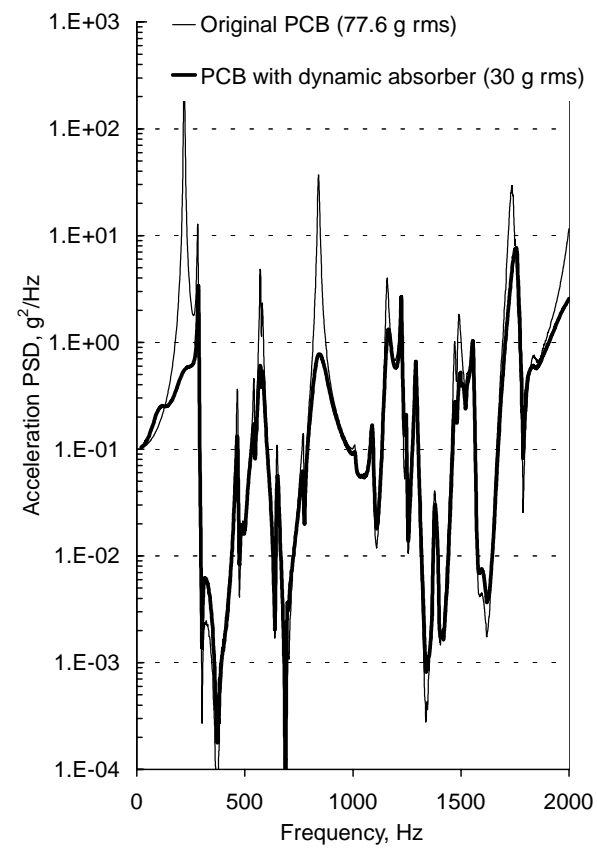

Point (3), suppression factor 2.6

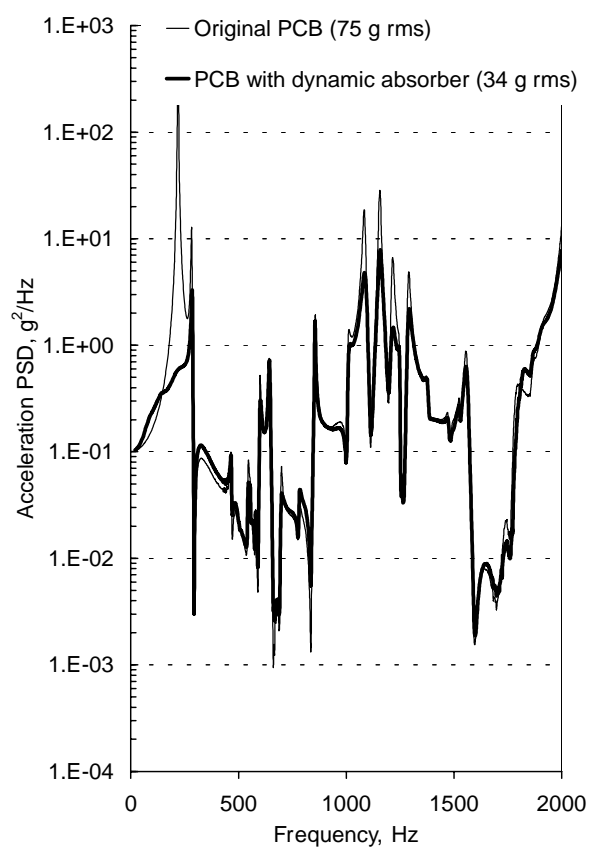

Point (2), suppression factor 2.2

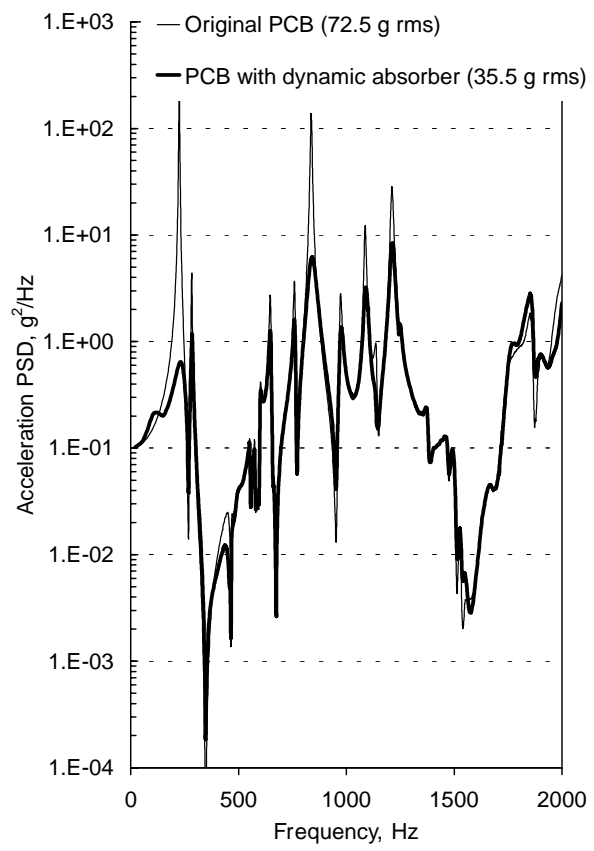

Point (4), suppression factor 2

Fig. 9. Performance of dynamic absorber at different points of PCB.

Fig. 10(b), using the dynamic absorber allows a 2-fold reduction in the peak-to-peak value of absolute acceleration and drastically relieves the entire transient process.
Further, the vibration controller was programmed to reproduce the typical swept sine test $10 \mathrm{~g} @ 10-500 \mathrm{~Hz}$, per [3]. The sweep rate was $0.5 \mathrm{~Hz} / \mathrm{s}$. Figure 11 shows the superimposed magnitude envelopes of the 


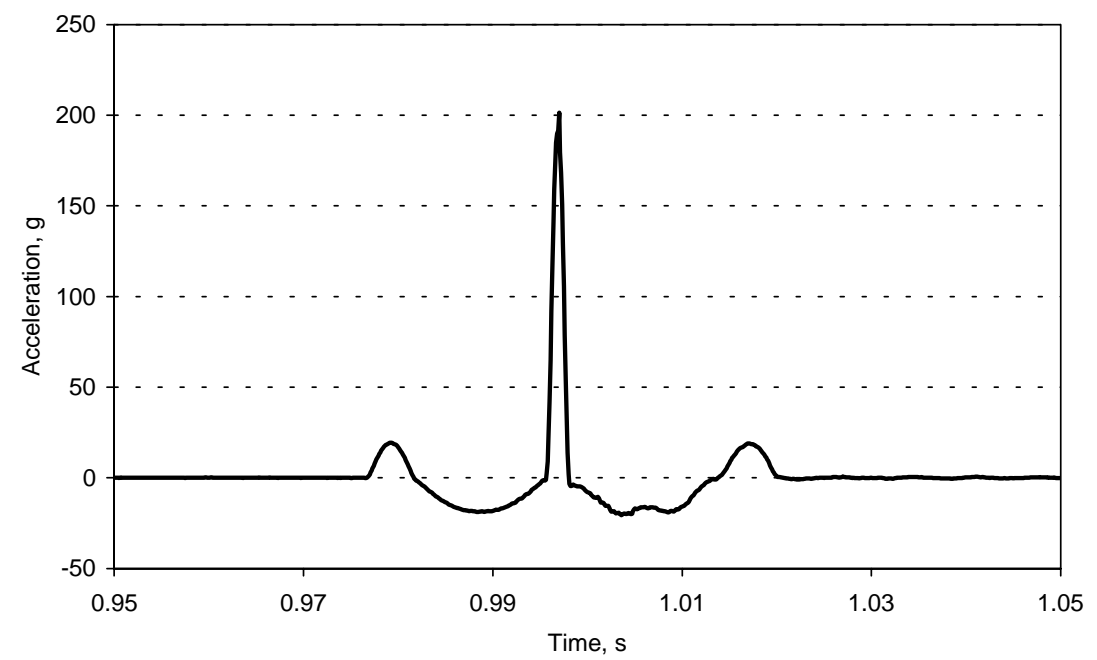

(a)

Time history of the shaker acceleration (half sine shock $200 \mathrm{~g} @ 3 \mathrm{~ms}$ )

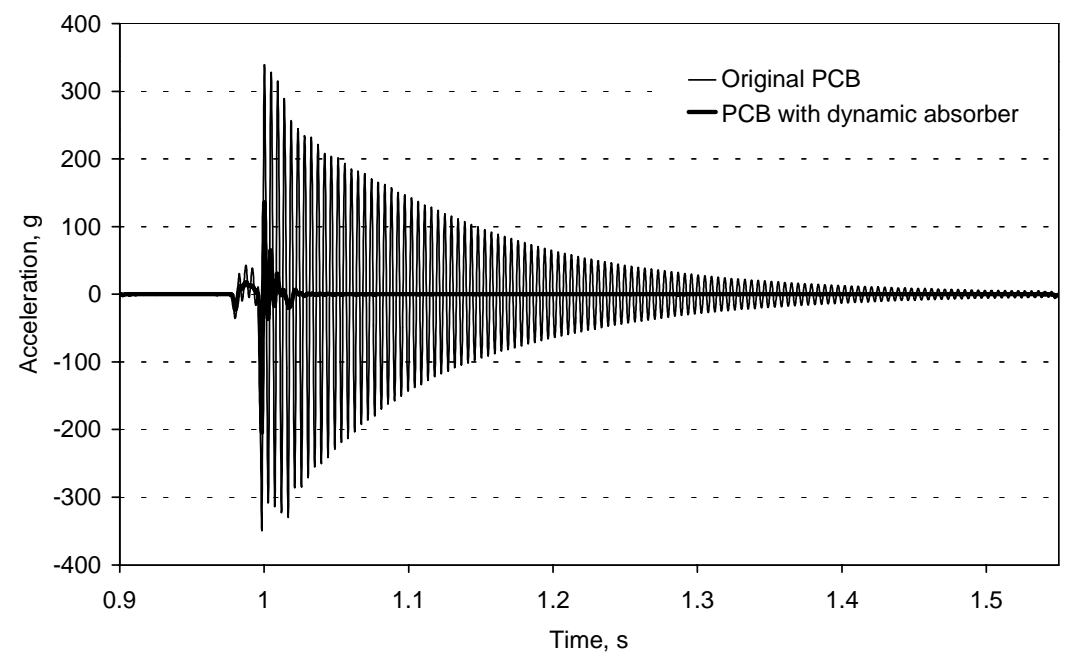

(b)

Time histories of the critical point of the original and modified PCB under shock excitation

Fig. 10. Performance of dynamic absorber under shock excitation. a) Time history of the shaker acceleration (half sine shock $200 \mathrm{~g} @ 3$ ms); b) Time histories of the critical point of the original and modified PCB under shock excitation.

dynamic responses measured at the critical point of the PCB before and after mounting the dynamic absorber. From Fig. 11, the resonant peak in the above dynamic response undergoes an 18-fold suppression.

\section{Conclusion}

The authors have shown theoretically and experimentally that the damped dynamic absorber can essentially suppress the dynamic responses and, therefore, increase the life of sensitive commercial-off-the-shelf PCBs operating in harsh environmental conditions under shock, wideband random and swept sine vibration. The novel technique developed by the authors allows the entire procedure of dynamic absorber optimisation to be simplified by avoiding the need for studying the dynamic model of the PCB and excitation factors.

Implementation of the damped dynamic absorber does not require a drastic increase in mass and dimensions, modification or redesign of commercial-offthe-shelf PCBs along with their mounting configura- 


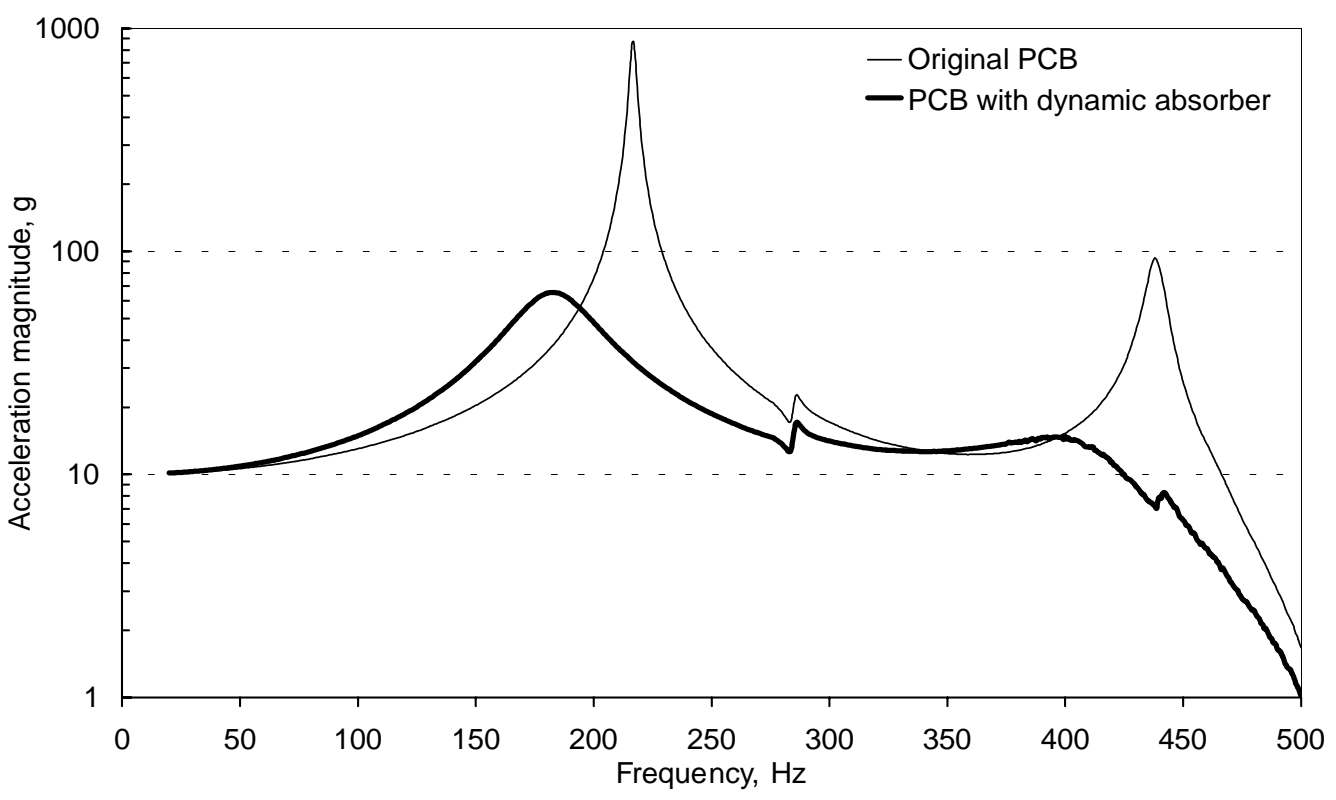

Fig. 11. Performance of dynamic absorber under swept sine excitation.

tion, and might be thought of as a prospective alternative/supplement to the existing methods of increasing reliability of sensitive electronic equipment. Further efforts should be aimed at developing compact, cheap and easily tuneable dynamic absorbers which maintain consistent properties over a wide range of ambient temperatures and lifetime.

\section{References}

[1] E. Jasson, Shock and Vibration Isolator for COTS equipment, COTS Journal (2000), http://www.rtcgroup.com/cotsjournal.

[2] D.S. Steingberg, Vibration analysis for electronic equipment, John Wiley and Sons, Inc., New York, 2000.

[3] Military Standard, Environmental Test Methods, MIL-STD810E 1988 Department of Defence, Washington, DC.

[4] Shock and Vibration, Design Tutorial, Chassis Plans Inc., http: //www.chassis-plans.com/html/shock_and_vibration.html.

[5] C. Stahle, Pioneering Damping in Space, Proceeding of Damping 89. Flight Dynamic Laboratory of Air Force Wright Aeronautical Laboratories, 1989.

[6] J.C. Snowdon, Vibration and Shock in Damped Mechanical System, John Wiley and Sons, Inc., New York, 1977.

[7] I. Straznicky, Designing Harsher Environment Circuit Boards, COTS Journal (Nov/Dec 2000), http://www.rtcgroup.com/ cotsjournal.

[8] V. Rogov and B. Davison, Vibration Test and Analysis, Test \& Screening, COTS journal March 2001, http://www.rtcgroup.com/cotsjournal.

[9] S.L. Malfa, P.A.A. Laura, C.A. Lrossit and O. Alvarez, Use of dynamic absorber in the case of a vibrating printed circuit board of complicated boundary shape, Journal of Sound and Vibration 230 (2000), 721-724.
[10] J. Starr and W. Tustin, What results from vibrating electronic systems, COTS Journal (April 2002), 37-42, http://www.rtcgroup.com/cotsjournal.

[11] J. Ormondroyd, J.P. Den Hartog, Theory of the dynamic absorber, Transaction of ASME $\mathbf{5 0}$ (1928), 9-22.

[12] G.B. Warburton and E.O. Ayorinde, Optimal absorber parameters for simple system, Earthquake Engineering and Structure Dynamic 8 (1980), 197-217.

[13] V.I. Babitsky, Dynamic absorption of vibration, in: Vibration and Impact Protection, K.V. Frolov, ed., Vibration in Engineering, (Vol. 6), Mashinostroenie, Moscow, 1981, (in Russian).

[14] Y.Z. Wang and S.H. Cheng, The optimal design of dynamic absorber in the time domain and the frequency domain, Applied Acoustic 28 (1989), 67-78.

[15] B.G. Korenev and L. Resnikov, Dynamic Vibration Absorber: Theory and Technical Application, John Wiley and Sons, Inc., Chichester, 1993.

[16] H. Nishimura, K. Yoshida and T. Shimogo, Optimal dynamic vibration absorber for multi-degree-of-freedom system (Theoretical consideration in the case of random input), JSME International Journal 32 (1989), 373-379.

[17] L. Kitis, P.B. Wang and W.D. Pilkey, Vibration reduction over a wide frequency range, Journal of Sound and Vibration $\mathbf{8 9}$ (1983), 559-569.

[18] A.R. Domingos and S. Valder, Optimisation of Dynamic Vibration Absorber Over a Frequency Band, Mechanical System and Signal Processing 14 (2000), 679-690.

[19] S.J. Bendat and A.G. Piersol, Random data: analysis and measurement procedures, Wiley Interscience, New York, 1986.

[20] M. Gertel, Specification of laboratory tests, in: Shock and Vibration Handbook, (Vol. 2), (No. 24), C.M. Harris and C.E. Crefe, eds, Mc Graw-Hill, 1961.

[21] R.H. Jacobson, Vibration and shock evaluation of airborne electronic component parts end equipment, WADC Technical Report 56-301, ASTIA-AD 123 658, 1956. 
[22] G.J. Hasslacher and H.L. Murray, Determination of an optimum vibration acceptance test, Shock, Vibration and Associated Environments Bulletin, Part III, No. 33, 1964, pp. 183188.
[23] C. Lalanne, Mechanical Vibration and Shock, Volume IV Fatigue damage, Hermes Penton Science, 2002.

[24] V.V. Bolotin, Random vibration of elastic systems, Martinces Nishoff Publishers, The Hague, 1984. 

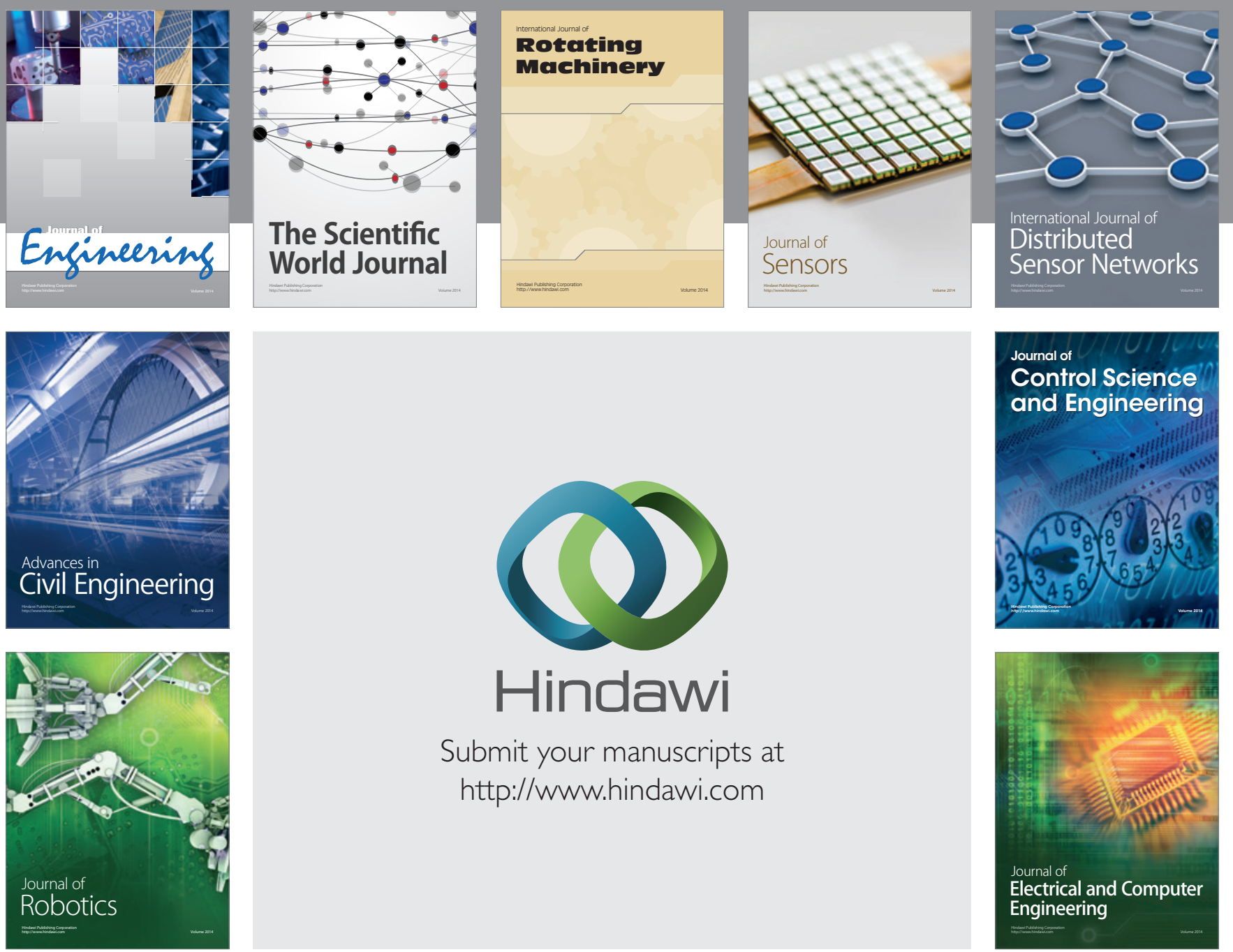

Submit your manuscripts at

http://www.hindawi.com
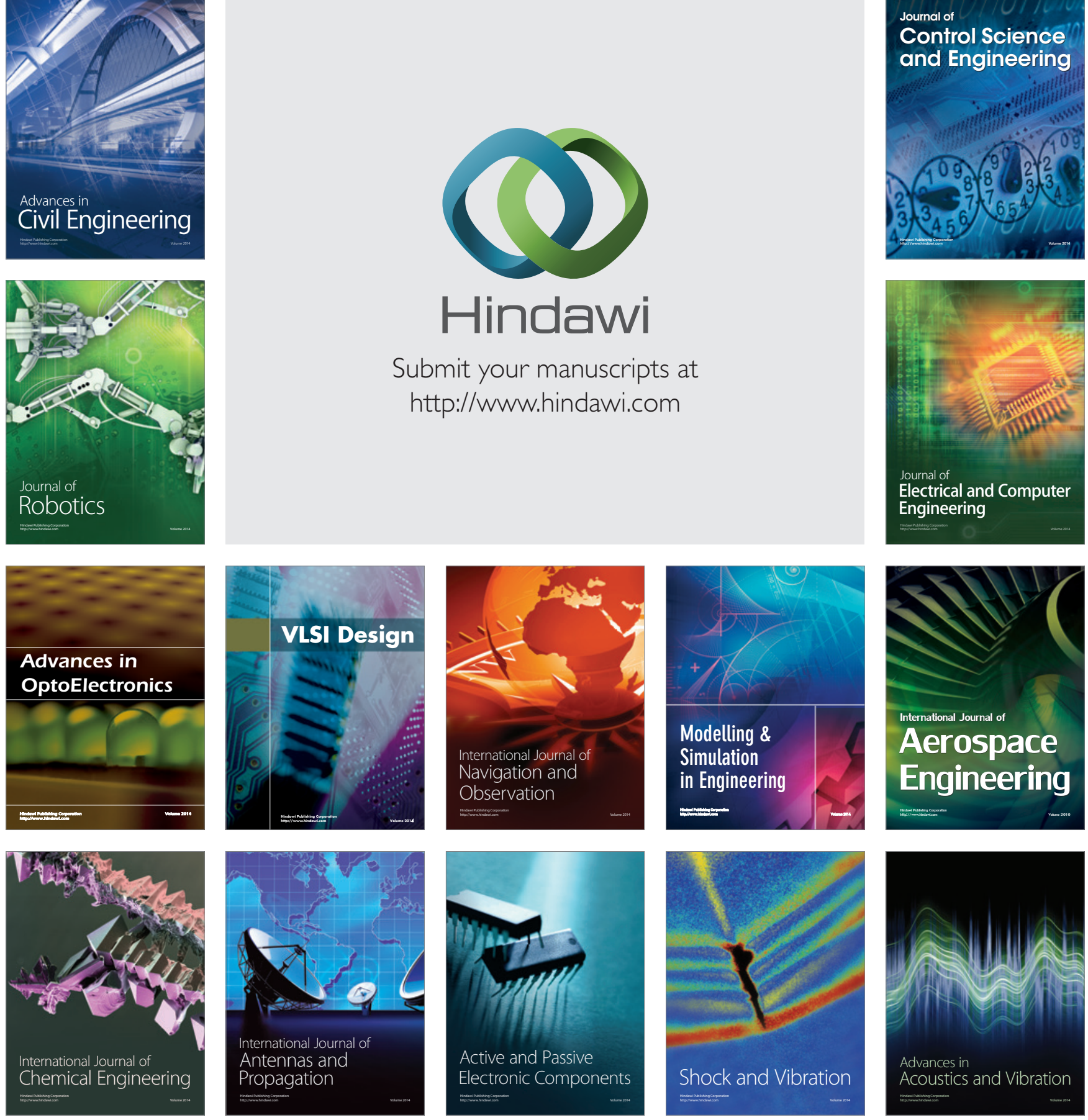